\title{
Agreement of self-reported physician diagnosis of migraine with international classification of headache disorders-II migraine diagnostic criteria in a cross-sectional study of pregnant women
}

\author{
Chunfang Qiu*, Michelle A Williams ${ }^{1,2}$, Sheena K Aurora ${ }^{3}$, B Lee Peterlin ${ }^{4}$, Bizu Gelaye ${ }^{2}$, Ihunnaya O Frederick
} and Daniel A Enquobahrie ${ }^{1,5}$

\begin{abstract}
Background: Migraine, a common chronic-intermittent disorder among reproductive age women, has emerged as a novel risk factor for adverse perinatal outcomes. Diagnostic reliability of self-report of physician-diagnosed migraine has not been investigated in pregnancy cohort studies. We investigated agreement of self-report of physician-diagnosed migraine with the diagnostic criteria promoted by the International Classification of Headache Disorders, 2nd edition (ICHD-II).

Methods: The cross-sectional study was conducted among 500 women who provided information on a detailed migraine questionnaire that allowed us to apply all ICHD-II diagnostic criteria.

Results: Approximately $92 \%$ of women reporting a diagnosis of migraine had the diagnosis between the ages of 11 and 40 years (<10 years 6.8\%; $11-20$ years $38.8 \%$; $21-30$ years $42.7 \% ; 31-40$ years $10.7 \%$; and $>40$ years $1.0 \%$ ). We confirmed self-reported migraine in $81.6 \%$ of women when applying the ICHD-II criteria for definitive migraine (63.1\%) and probable migraine (18.5\%).
\end{abstract}

Conclusion: There is good agreement between self-reported migraine and ICHD-II-based migraine classification in this pregnancy cohort. We demonstrate the feasibility of using questionnaire-based migraine assessment according to full ICHD-II criteria in epidemiological studies of pregnant women.

Keywords: Migraine, Pregnancy, Diagnosis, ICHD-II, Self-report, Agreement

\section{Background}

Migraine, a common chronic-intermittent neurovascular headache disorder, is ranked among the world's twenty most disabling medical conditions by the World Health Organization [1]. Migraine is characterized by episodic severe headache accompanied by autonomic nervous system dysfunction. Women are more commonly affected than men, with reported lifetime prevalence estimates of $16-32 \%$ for women and 6-9\% for men [2-6]. Migraine risk varies considerably across the life course. The prevalence of migraine in women rises after the average age of

\footnotetext{
* Correspondence: Chun-fang.Qiu@Swedish.org

${ }^{1}$ Center for Perinatal Studies, Swedish Medical Center, 1124 Columbia Street, Suite 750, Seattle, WA 98104, USA

Full list of author information is available at the end of the article
}

menarche, and peaks before the average age of menopause [7]. Thus migraines are most prevalent among women in their childbearing years.

Migraine has emerged as a novel risk factor for adverse perinatal outcomes including hypertensive disorders of pregnancy [8,9], preterm birth [10] and placental abruption [11]. Most prior epidemiologic studies have relied on self-report of physician-diagnosed migraine as a means for classifying pregnant women with a history of migraine. Data from the Women's Health Study (a study of women aged $\geq 45$ years) showed very good agreement between self-reported migraine and ICHD-II-based diagnosed migraine. The investigators confirmed self-reported migraine in $>87 \%$ of women when applying the International Classification of Headache Disorders 2004 criteria (ICHD-II) 
for definitive migraine $(71.5 \%)$ and probable migraine (16.2\%) without aura [12]. We are unaware of studies that investigated diagnostic reliability (commonly measured using inter-rater agreement) of self-report of physiciandiagnosed migraine in pregnancy cohort studies.

In a cross-sectional study, we investigated 500 pregnant women who provided information on a detailed migraine questionnaire that allowed us to apply ICHD-II diagnostic criteria, promoted by International Headache Society (IHS), and examined the agreement of self-reported physician diagnosis of migraine with migraine determined using the ICHD-II diagnostic criteria ("golden standard") in this report.

\section{Methods}

Study participants were pregnant women attending prenatal care at clinics affiliated with Swedish Medical Center in Seattle, Washington and enrolled in the Migraine and Pregnancy Study, a pregnancy cohort study designed to investigate the relationship between migraine, headache symptoms before and during pregnancy, and the risk of preeclampsia [13]. The study population for this report is from the first 500 participants who were enrolled (consecutively) and were interviewed during the period of April 2009 and December 2010. Women were ineligible if they initiated prenatal care after 20 weeks gestation, were younger than 18 years of age, did not speak and read English, did not plan to carry the pregnancy to term, or did not plan to deliver at Swedish Medical Center. Participants completed a questionnaire administered by trained interviewers (supervised by neurologist and maternal fetal medicine clinicians) at enrollment. Participants were asked to provide information pertaining to their medical history, pre-pregnancy weight, general health, pregnancy-related symptoms, socio-demographic, and lifestyle characteristics. The interview included a structured migraine assessment questionnaire (adapted from the deCODE Genetics migraine questionnaire (DMQ3) [14] (Additional file 1) and an assessment of disability associated with headaches experienced before and during pregnancy by Migraine Disability Assessment (MIDAS) Questionnaire [15]. In previous validation study, using a physician-conducted interview as an empirical index of validity, the deCODE Migraine Questionnaire (DMQ3) diagnosed migraine with a sensitivity of 99\%, a specificity of $86 \%$ and a kappa statistic of 0.89 [16]. The detailed migraine-specific questionnaire contained questions addressing age at migraine onset, physician diagnosis of migraine, family history of migraine, details about migraine attacks and medication used.

Headache classification was determined using the ICHDII criteria established by the International Headache Society (IHS) [17]. "Definitive Migraine" (IHS category 1.1 or 1.2) was defined by at least five lifetime headache attacks (criterion A) lasting 4-72 hours (criterion B), with at least two of the qualifying pain characteristics [unilateral location (criterion C1), pulsating quality (criterion C2), moderate or severe pain intensity (criterion C3), aggravation by routine physical exertion (criterion C4)]; at least one of the associated symptoms [nausea and/or vomiting (criterion D1), photo/phonophobia (criterion D2)]; and not readily attributable to another central nervous system disorder or head trauma (according to subject self-report) (criterion E). "Probable Migraine" (IHS category 1.6) was designated if all but one of the definitive migraine criteria were fulfilled, excluding headaches attributable to another disorder. Finally, any migraine was defined as the group with either definitive migraine or probable migraine combined.

The procedures used in the study were in agreement with the protocol approved by the Institutional Review Board of Swedish Medical Center (Swedish IRB \# 008567). All participants provided written informed consent.

Frequency distributions of sociodemographic, reproductive, medical and behavioral factors among groups defined by ICHD-II (any migraine, no migraine) and the cohort were compared using means ( \pm standard deviation (SD)) for continuous variables and counts and percentages for categorical variables. Bivariate differences in characteristics associated with definitive and probable migraine headaches were determined using Chi-square test (or Fisher's exact test) for categorical variables and Student's $t$-test for continuous variables.

The self-reported physician migraine diagnosis was compared with the ICHD-II based diagnosis. The sensitivity and specificity as well as positive predictive value and negative predictive value of the self-reported diagnosis were assessed. Concordance also was determined by estimating the value of Cohen's kappa coefficient [18]. All analyses were performed using Stata 9.0 (Stata, College Station, TX) statistical analysis software. All reported p-values are two-tailed. The $95 \%$ confidence interval (CI) for the prevalence estimate of migraine was determined using previously described methods [19].

\section{Results}

A total of 100 subjects met the ICHD-II criteria for definitive migraine, and 49 additional subjects met the criteria for probable migraine. The lifetime prevalence of definitive migraine was $20.0 \%$ (95\% CI 16.6-23.8\%). When probable migraine was included, the lifetime prevalence of migraine in this population increased to $29.8 \%$ (95\% CI 25.9-34.0\%). However, chronic migraine, defined as headache that occurs 15 or more days a month with headache lasting 4 hours or longer for at least 3 consecutive months in individuals with current or prior diagnosis of migraine, occurred only in 12 women out of 149 ICHD-II diagnosed migraine cases. 
Characteristics of participants according to ICHD-IIdefined migraine status are presented in Table 1 . A positive family history of migraine (defined as report of migraine for at least one of the following relatives: father, mother, sibling, grandparents, children) was reported by $65.1 \%$ of participants with migraine and by $35.0 \%$ of individuals without migraine. Participants with migraine tended to have higher pre-pregnancy body mass index and were more likely to report a positive history of chronic hypertension.

We confirmed self-reported migraine in $81.6 \%$ of women when applying the ICHD-II criteria for definitive migraine (63.1\%) and probable migraine (18.5\%) (Table 2). Overall, when compared to the ICHD-II diagnosis, the self-reported physician diagnosis had a sensitivity of $56.8 \%$ (95\% CI
48.7\%-64.5\%); a specificity of 94.6\% (95\% CI 91.7\%-96.5\%) and positive predictive value of $81.6 \%$ (95\% CI $73.0 \%$ $87.9 \%$ ), as well as negative predictive value of $83.8 \%$ (95\% CI 79.8\%-87.1\%). Cohen's kappa coefficient was 0.56 . We did not observe differences in Choen's kappa coefficients when analyses were stratified by parity (i.e., nulliparous vs. multiparous). Characteristics such as nausea and/or vomiting and photophobia were far more prevalent among women who reported having a physician diagnosis of migraine as compared with their counterparts without such a diagnosis. Approximately $92 \%$ of women reporting a diagnosis of migraine had the diagnosis between the ages of 11 and 40 years $(<10$ years $6.8 \%$; $11-20$ years $38.8 \%$; $21-30$ years $42.7 \%$; $31-40$ years 10.7\%; and $>40$ years $1.0 \%$ ) (Figure 1 ).

Table 1 Maternal characteristics of study population according to ICHD-II criteria migraine (definitive + probable), April 2009-Dec 2010, Seattle, WA

\begin{tabular}{|c|c|c|c|c|}
\hline \multirow[t]{2}{*}{ Characteristics } & \multirow{2}{*}{$\begin{array}{c}\text { Cohort } \\
(\mathrm{N}=500)\end{array}$} & \multicolumn{3}{|c|}{ ICHD-II criteria for migraine (definitive + probable) } \\
\hline & & Yes $(N=149)$ & No $(N=351)$ & $\mathrm{p}$-value \\
\hline Maternal Age (years) & $33.4 \pm 4.2$ & $32.9 \pm 4.4$ & $33.6 \pm 4.1$ & 0.131 \\
\hline \multicolumn{5}{|l|}{ Maternal Age (years) } \\
\hline$<35$ & $316(63.2)$ & $102(68.5)$ & $214(61.0)$ & \multirow[t]{2}{*}{0.112} \\
\hline$\geq 35$ & $184(36.8)$ & $47(31.5)$ & $137(39.0)$ & \\
\hline \multicolumn{5}{|l|}{ Maternal Race/Ethnicity } \\
\hline Non-Hispanic White & $430(86.0)$ & $126(84.6)$ & $304(86.6)$ & \multirow[t]{4}{*}{0.593} \\
\hline African American & $8(1.6)$ & $4(2.7)$ & $4(1.1)$ & \\
\hline Other & $57(11.4)$ & $18(12.1)$ & $39(11.1)$ & \\
\hline Missing & $5(1.0)$ & $1(0.7)$ & $4(1.1)$ & \\
\hline \multicolumn{5}{|l|}{ Annual Household Income (\$) } \\
\hline$<50,000$ & $20(4.0)$ & $6(4.0)$ & $14(4.0)$ & \multirow[t]{4}{*}{0.061} \\
\hline $50,000-69,000$ & $33(6.6)$ & $13(8.7)$ & $20(5.7)$ & \\
\hline$\geq 70,000$ & $428(85.6)$ & $129(86.6)$ & $299(85.2)$ & \\
\hline Missing & $19(3.8)$ & $1(0.7)$ & $18(5.1)$ & \\
\hline Single Marital Status & $48(9.6)$ & $19(12.8)$ & $29(8.3)$ & 0.119 \\
\hline Nulliparous & $261(52.2)$ & $81(54.4)$ & $180(51.3)$ & 0.528 \\
\hline Unplanned Pregnancy & $72(14.4)$ & $24(16.1)$ & $48(13.7)$ & 0.479 \\
\hline Cigarette Smoker during early pregnancy & $95(19.0)$ & $30(20.1)$ & $65(18.5)$ & 0.674 \\
\hline Family History of Headache/Migraine & $220(44.0)$ & $97(65.1)$ & $123(35.0)$ & $<0.001$ \\
\hline History of Chronic Hypertension & $11(2.2)$ & $7(4.7)$ & $4(1.1)$ & 0.020 \\
\hline Pre-Pregnancy Body Mass Index $\left(\mathrm{kg} / \mathrm{m}^{2}\right)$ & $23.5 \pm 4.7$ & $24.7 \pm 5.4$ & $23.0 \pm 4.3$ & $<0.001$ \\
\hline \multicolumn{5}{|l|}{ Pre-Pregnancy Body Mass Index (kg/m²) } \\
\hline$<18.5$ & $24(4.8)$ & $5(3.4)$ & $19(5.4)$ & \multirow[t]{5}{*}{0.012} \\
\hline $18.5-24.9$ & $340(68.0)$ & 89 (59.7) & 251 (71.5) & \\
\hline $25.0-29.9$ & $92(18.4)$ & $34(22.8)$ & $58(16.5)$ & \\
\hline$\geq 30.0$ & $42(8.4)$ & $20(13.4)$ & $22(6.3)$ & \\
\hline Missing & $2(0.4)$ & $1(0.7)$ & $1(0.3)$ & \\
\hline
\end{tabular}

Data in mean \pm standard deviation (SD) or number (\%). 
Table 2 Summary of self-reported migraine and ICHD-II classified migraine

\begin{tabular}{|c|c|c|}
\hline \multirow[t]{3}{*}{ Characteristics } & \multicolumn{2}{|c|}{ Self-reported migraine } \\
\hline & Yes & No \\
\hline & $(\mathrm{N}=103)$ & $(\mathrm{N}=395)$ \\
\hline \multicolumn{3}{|l|}{ ICHD-II Diagnostic Criteria: Migraine } \\
\hline No & 19 (18.5) & $331(83.8)$ \\
\hline Yes & $84(81.6)$ & $64(16.2)$ \\
\hline ICHD-II criteria migraine (probable) & $19(18.5)$ & $29(7.3)$ \\
\hline ICHD-II criteria migraine (definitive) & $65(63.1)$ & $35(8.9)$ \\
\hline Lifetime number of headache/migraine attacks $\geq 5$ (criterion A) & $95(92.2)$ & $94(23.8)$ \\
\hline Attacks lasting 4-72 hours (criterion B) & $80(77.7)$ & $73(18.5)$ \\
\hline Unilateral headache location (criterion C1) & $59(57.3)$ & $43(10.9)$ \\
\hline Pulsating pain quality (criterion C2) & $62(60.2)$ & $60(15.2)$ \\
\hline Moderate or severe pain intensity (criterion C3) & $95(92.2)$ & $101(25.6)$ \\
\hline Aggravation by routine physical activity (criterion C4) & $72(69.9)$ & $70(17.7)$ \\
\hline Nausea and/or vomiting (criterion D1) & $80(77.7)$ & $64(16.2)$ \\
\hline Photophobia & $91(88.4)$ & $90(22.8)$ \\
\hline Phonophobia & $86(83.5)$ & $79(20.0)$ \\
\hline Photo- and phonophobia (criterion D2) & 82 (79.6) & 66 (16.7) \\
\hline
\end{tabular}

2 subjects missing self-reported migraine information. Data in number (\%).

ICHD-II, International Classification of Headache Disorders, version 2 (ICHD-II).

\section{Discussion}

Our study finding of a high prevalence (29.8\%) of migraine in this cohort of pregnant women of reproductive age is consistent with prior literature [3-5]. For example, the American Migraine Prevalence and Prevention (AMPP) Study found that the highest 1-year period prevalence was observed among those ages 18-59. In that cohort, $17.1 \%$ of women met diagnostic criteria for definitive migraine; and additional 5.1\% met diagnostic criteria for probable migraine [5]. Our study also showed good agreement between self-reported migraine and ICHD-

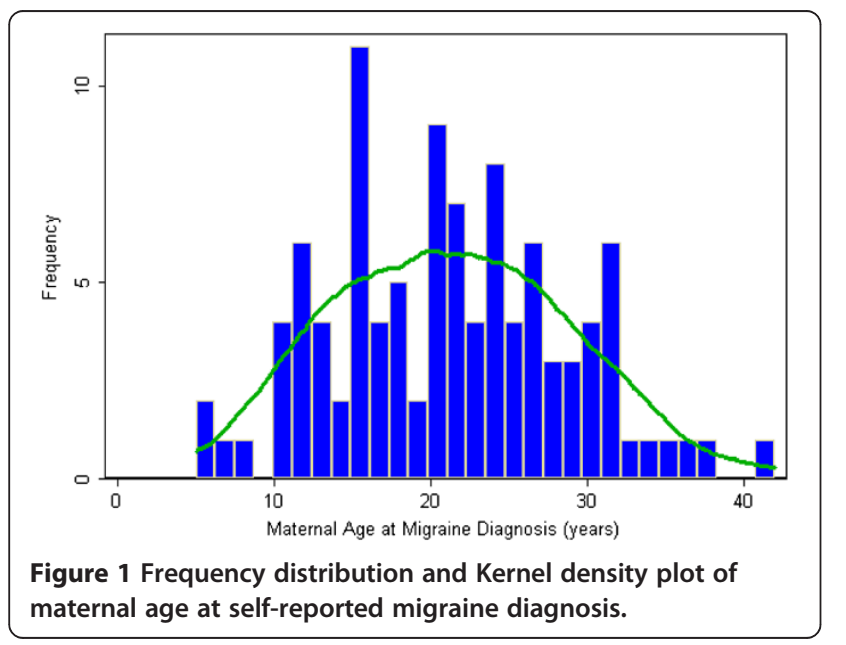

II-based migraine classification. Our results were consistent with reports by Schurks et al., who reported good agreement between self-reported migraine and ICHD-II defined migraine in a cohort of non-pregnant women [12]. Since migraine diagnosis relies on the presence of certain symptoms as well as associated features, information that can easily be obtained using questionnaires, the agreement of such self-reports with the ICHD-II criteria is crucial for studying migraine-specific characteristics in populationbased epidemiological studies.

The strengths of our study include the relatively large number of women with information from the detailed standardized questionnaire allowing us to apply all ICHDII criteria for migraine, and the opportunity to compare with self-reported "physician-diagnosed" migraine information. To our best knowledge, this is the first study that investigated the agreement of self-reported migraine with ICHD-II diagnosis in a pregnancy cohort. Pregnancy is a unique condition. During the first trimester, women experience increased physiological and psychological changes such as hormonal surge and increased blood volume. Lack of sleep, low blood sugar, dehydration and possible caffeine withdrawal could also contribute to the occurrence of migraine attacks. The headache may be further aggravated by stress [20,21]. In addition, there is a heightened cautiousness among women with regards to using medication during early pregnancy to prevent or treat their headaches. Concurrently, most studies on 
migraine in pregnancy detected a decrease in frequency of reported migraine attacks and pain intensity in the 2nd and 3rd trimesters as hormone levels stabilize [22-24].

Some important limitations must be considered when interpreting the results of our study. First, our ICHD-IIdiagnosed migraine is based on questionnaires administered by trained interviewers instead of the gold standard of physician examination. However, it is not feasible to take implement full scale physical examinations with medical histories in large epidemiological studies, making our approach a practical alternative. In this study, we observed good agreement between ICHD-II diagnosed migraine and self-reported "physician diagnosed" migraine. Secondly, misclassification of headache type is possible. The questionnaire did not ask women to describe multiple headache types, and it is presumed that subjects described the headache episodes most burdensome to them. Misunderstanding one or more questions on the migraine-specific questionnaire may also lead to a misclassification according to the ICHD-II criteria. Third, caution must be taken that a diagnosis made during pregnancy may be attributable to transient changes in the characters of primitive headaches [25]; however, in the current study, 93.3\% of ICHD-II defined migraine patients reported that their headache attacks started more than one year before the interview, hence mitigating concern of misclassification in this case. Finally, in this study, only a small minority of women with migraine were classified as having chronic migraine per se. Recall bias is possible although migraines are usually dramatic events well remembered by the affected persons.

\section{Conclusions}

There is good agreement between self-reported physiciandiagnosed migraine and ICHD-II-based migraine classification in this pregnancy cohort. Our findings demonstrate the feasibility of using questionnaire-based migraine assessment according to full ICHD-II criteria in epidemiological studies of pregnant women.

\section{Additional file}

Additional file 1: The deCODE Genetics migraine questionnaire (DMQ3).

\section{Abbreviations}

ICHD-II: The International Classification of Headache Disorders 2004 criteria, version II; IHS: International Headache Society; SD: Standard deviation; 95\% CI: 95\% confidence interval; AMPP: American Migraine Prevalence and Prevention.

\section{Competing interests}

The authors declare that they have no competing interests.

\section{Authors' contributions}

MAW acquired funding for the study and developed the study design. SKA and BLP helped develop the study design. IOF monitored the data collection. CQ, DAE and MAW developed the analytical plan; CQ completed the statistical analysis. CQ, DAE, MAW drafted the manuscript. All authors edited the manuscript. All authors read and approved the final manuscript.

\section{Acknowledgment}

This research was supported by an award from the National Institutes of Health (R01HD-055566). The authors are indebted to the staff of the Center for Perinatal Studies for their expert technical assistance.

\section{Author details}

${ }^{1}$ Center for Perinatal Studies, Swedish Medical Center, 1124 Columbia Street, Suite 750, Seattle, WA 98104, USA. ${ }^{2}$ Department of Epidemiology, Harvard School of Public Health, Boston, MA, USA. ${ }^{3}$ Department of Neurology, Stanford University, Stanford, California, USA. ${ }^{4}$ Johns Hopkins University School of Medicine, Baltimore, MD, USA. ${ }^{5}$ Department of Epidemiology, School of Public Health, University of Washington, Seattle, WA, USA.

Received: 18 July 2013 Accepted: 4 December 2013

Published: 13 December 2013

\section{References}

1. World Health Organization: Headache disorders. http://www.who.int/ mediacentre/factsheets/fs277/en/ (accessed on April 2013).

2. Steiner TJ, Scher Al, Stewart WF, Kolodner K, Liberman J, Lipton RB: The prevalence and disability burden of adult migraine in England and their relationships to age, gender and ethnicity. Cephalalgia 2003, 23:519-527.

3. National health and nutrition examination survey website. Available at: http://www.cdc.gov/nchs/nhanes.htm (accessed December 17, 2012).

4. Kalaydjian A, Merikangas K: Physical and mental comorbidity of headache in a nationally representative sample of US adults. Psychosom Med 2008, 70:773-780.

5. Lipton RB, Bigal ME, Diamond M, Freitag F, Reed ML, Stewart WF, AMPP Advisory Group: Migraine prevalence, disease burden, and the need for preventive therapy. Neurology 2007, 68:343-349.

6. Smitherman TA, Burch R, Sheikh H, Loder E: The prevalence, impact, and treatment of migraine and severe headaches in the United States: a review of statistics from national surveillance studies. Headache 2013, 53:427-436.

7. Nappi RE, Berga SL: Migraine and reproductive life. Handb Clin Neurol 2010, 97:303-322.

8. Simbar M, Karimian Z, Afrakhteh M, Akbarzadeh A, Kouchaki E: Increased risk of pre-eclampsia (PE) among women with the history of migraine. Clin Exp Hypertens 2010, 32:159-165.

9. Williams MA, Peterlin BL, Gelaye B, Enquobahrie DA, Miller RS, Aurora SK: Trimester-specific blood pressure levels and hypertensive disorders among pregnant migraineurs. Headache 2011, 51:1468-1482.

10. Blair EM, Nelson KB: Migraine and preterm birth. J Perinatol 2011, 31:434-439.

11. Sanchez SE, Williams MA, Pacora PN, Ananth CV, Qiu C, Aurora SK, Sorensen TK: Risk of placental abruption in relation to migraines and headaches. BMC Womens Health 2010, 10:30.

12. Schürks $M$, Buring JE, Kurth $T$ : Agreement of self-reported migraine with ICHD-II criteria in the Women's Health Study. Cephalalgia 2009, 29:1086-1090.

13. Frederick IO, Qiu C, Enquobahrie DA, Aurora SK, Peterlin BL, Gelaye B, Williams MA: Lifetime prevalence and correlates of migraine among women in a pacific northwest pregnancy cohort study. Headache 2013. Epub.

14. deCODE Genetics. http://www.decode.com/migraine/questionnaire (for the English translation of the Icelandic, deCODE migraine questionnaire used in this study).

15. Stewart WF, Lipton RB, Dowson AJ, Sawyer J: Development and testing of the Migraine Disability Assessment (MIDAS) Questionnaire to assess headache-related disability. Neurology 2001, 56:S20-S28.

16. Kirchmann M, Seven E, Björnsson A, Björnssdóttir G, Gulcher JR, Stefánsson $K$, Olesen J: Validation of the deCODE Migraine Questionnaire (DMQ3) for use in genetic studies. Eur J Neurol 2006, 13:1239-1244.

17. Headache Classification Subcommittee of the International Headache Society: The international classification of headache disorders, 2nd edition. Cephalalgia 2004, 44(Suppl 1):S5-S12.

18. Cohen J: A coefficient of agreement for nominal scales. Education Psychol Measure 1960, 20:37-46.

19. Colton T: Statistics in medicine. Boston: Little, Brown and Company; 1974:P160

20. Andress-Rothrock D, King W, Rothrock J: An analysis of migraine triggers in a clinic-based population. Headache 2010, 50:1366-1370. 
21. Milde-Busch A, Blaschek A, Heinen F, Borggräfe I, Koerte I, Straube A, Schankin C, von Kries R: Associations between stress and migraine and tension-type headache: results from a school-based study in adolescents from grammar schools in Germany. Cephalalgia 2011, 31:774-785.

22. Chen $T C$, Leviton $A$ : Headache recurrence in pregnant women with migraine. Headache 1994, 34:107-110.

23. Scharff $L$, Marcus DA, Turk DC: Headache during pregnancy and in the postpartum: a prospective study. Headache 1997, 37:203-210.

24. Kvisvik EV, Stovner $\sqcup$, Helde G, Bovim G, Linde M: Headache and migraine during pregnancy and puerperium: the MIGRA-study. J Headache Pain 2011, 12:443-451.

25. Maggioni F, Alessi C, Maggino T, Zanchin G: Headache during pregnancy. Cephalalgia 1997, 17:756-759.

doi:10.1186/1472-6874-13-50

Cite this article as: Qiu et al: Agreement of self-reported physician diagnosis of migraine with international classification of headache disorders-II migraine diagnostic criteria in a cross-sectional study of pregnant women. BMC Women's Health 2013 13:50.

\section{Submit your next manuscript to BioMed Central and take full advantage of:}

- Convenient online submission

- Thorough peer review

- No space constraints or color figure charges

- Immediate publication on acceptance

- Inclusion in PubMed, CAS, Scopus and Google Scholar

- Research which is freely available for redistribution 